\title{
Hypothesis
}

\section{Elucidating hydrogenase surfaces and tracing the intramolecular tunnels for hydrogenase inhibition in microalgal species}

\author{
Kritika Dixit*, Md.Akhlaqur Rahman, Adi Nath \& Shanthy Sundaram
}

Centre of Biotechnology, University of Allahabad, Allahabad, Uttar Pradesh 211002 India; Kritika Dixit - E-mail: kritika.mhg.bhu10@gmail.com; *Corresponding author

\begin{abstract}
:
Intramolecular tunnels are majorly attracting attention as possible pathways for entry of inhibitors like oxygen and carbon monoxide to the active sites of the enzymes, hydrogenases. The results of homology modeling of the HydSL protein, a NiFe-hydrogenase from Chlamydomonas reinhardtii and Chlorella vulgaris are presented in this work. Here we identify and describe molecular tunnels observed in HydSL hydrogenase enzyme systems. The possible determinant of the oxygen stability of already studied hydrogenases could be the lack of several intramolecular tunnels. The possible tunnels were traced out using MOLE 2 software, which showed several intramolecular pathways that may be connecting the active sites of the enzyme. The RMSD value showed a great deal of significance in the enzyme homology. This is the first report of its kind in which mapping of the intramolecular tunnels in the four-hydrogenase enzymes disclosed potential variations between designed models and acknowledged structures. We are seeking out the explanations for oxygen sensitivity of studied hydrogenases within the structure of intramolecular tunnels. Local and Global RMSD (Root mean square deviation) was calculated for models and templates, which showed value of 1.284 indicating a successful homology model. The tunnel tracing study by Mole 2 indicated two tunnels joined into one in C. reinhardtii model whereas C. vulgaris model showed one tunnel almost like two tunnels. Templates of both the A. vinosum and D. vulgaris hydrogenase consisted of six tunnels. For HydSL from Chlamydomonas and Chlorella Species the maximal potential was set to $250 \mathrm{kcal} / \mathrm{mol}(1,046 \mathrm{~kJ} / \mathrm{mol})$ and the positive potential areas were marked. Electrostatic studies define electrostatic potential (ESP) that help shuttle protons to the active site.
\end{abstract}

Keywords: intramolecular tunnels; homology modeling; hydrogenases

Background:

[NiFe] hydrogenases are the most common and abundantly found hydrogenases in bacteria and archaea. Under physiological conditions, these enzymes are mostly found to catalyze $\mathrm{H}_{2}$ uptake. Contrary to what has been reported for $[\mathrm{Fe}]$ hydrogenases, the release of $\mathrm{H}_{2}$ is possible with [NiFe] hydrogenases in vitro as well. A variety of [NiFe] hydrogenase has been crystallized, all of these which originate from sulphatereducing bacteria. The periplasmic, membrane-bound hydrogenase of Desulfovibrio vulgaris is the best-studied member of the [NiFe] class [1]. The active site of [NiFe] hydrogenases consists of a nickel atom, sulphur surrounded by four cysteine residues. Two of these cysteines residues bridge to another iron atom. Nickel exhibits two sites or coordinates one "terminal" and one "bridging". The iron atom has been shown binding the three accepting ligands, which are two $\mathrm{CN}$ and one $\mathrm{CO}$ group. It shares the bridging coordination site with nickel (Figure 1). The complex $\mathrm{O}_{2}$ sensitivity of this class of hydrogenases is reviewed in this work. Earlier it was known that an enzyme had one active site but with molecular advances, researchers have found multiple active sites connected via molecular tunnels on a single enzyme unit, first being tryptophan. The apparent mechanisms rendered by these molecular conduits include the guarding of unstable intermediates and an improvement in catalysis by 


\section{Open access}

blocking the diffusion of the intermediates into the solvent. The translocation of the ammonia molecules, resulting from the hydrolysis of glutamine, is the most common functional essentiality of a protein tunnel that has been identified so far.

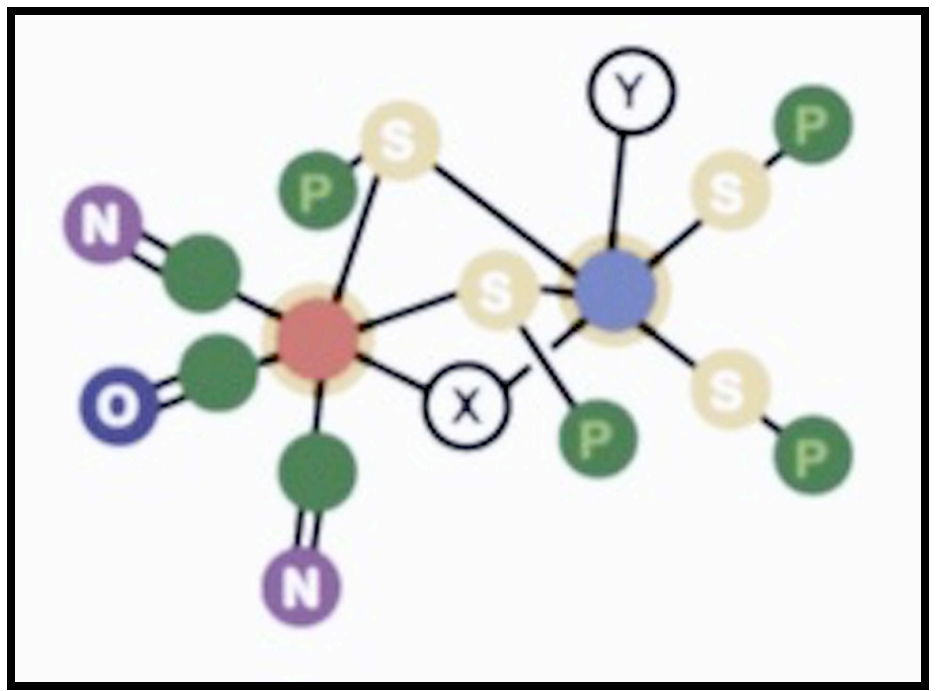

Figure 1: Typical [NiFe] prosthetic group-The nickel atom (light blue) is bound by four cysteine thiloates. Two of these residues bridge to the iron atom (red). The iron atom carries two $\mathrm{CN}$ and as single CO ligand. Furthermore, it shares a free coordination site $(X)$ with nickel. This bridging position is supposed to be the catalytic site. Nickel has another open coordination site $(Y)$ responsible where external $\mathrm{CO}$ inhibits the enzyme.

The first similarity model of HydSL from Clamydomonas reinhardtii was engineered by Szilagyi in 2002. During this work, X-ray structure of Desulfovibrio periplasmic hydrogenase was serving as a template (PDB entry ${ }_{2} \mathrm{FRV}$ ). The prosthetic teams of the protein were rather poorly present within the model (nickeliron site comprised solely two atoms, iron and nickel, without auxiliary ligand). Beside that, fifty-five C-terminal organic compound residues weren't enclosed into the model owing to the dearth of their homolog within the model. During this work, a proposal that an oversized range of helpful static interactions between subunits (ionic pairs) might be one among the explanations of high thermal stability of C. reinhardtii HydSL hydrogenase was put forward. The choice for this version is that the hypothesis on stabilization of the hydrogenase via oligomerization. NiFe-hydrogenase HydSL from Chlorella vulgaris was isolated to unvaried state comparatively recently [2]. It's similar in its properties to HydSL from $C$. reinhardtii: it maintained eighty four make the most initial activity after two hours incubation at $80^{\circ} \mathrm{C}$, and $100 \%$ activity when storage on air at $4^{\circ} \mathrm{C}$ for forty five days. This makes it as suitable candidate for application in hydrogen technologies as hydrogenase HydSL from C. reinhardtii. Beside similar chemical properties, these hydrogenases share common assembly mechanisms: heterologous expression of HydSL genes from C. reinhardtii with accessory genes from $C$. vulgaris led to the formation of useful product. The currently present models of this protein from Modbase information don't embody ligands, and that we couldn't notice them in alternative open sites. Each these enzymes drew attention as potential elements of photobiological hydrogen production systems owing to their high resistance to gas and therefore the ability to operate in phototrophic organisms. Since publication of the primary similarity model of $C$. reinhardtii hydrogenase, several alternative nickel-iron hydrogenases are with success crystallized, and their 3D structures are revealed. This allowed us to settle on a lot of appropriate model for similarity modeling.

Beside that, several powerful machine programs capable of reliable super molecular structure prediction became obtainable. The goal of this work was modeling HydSL hydrogenases from C. reinhardtii and C. vulgaris deep ecotype as well as ligands and highest range of organic compound residues, estimation of accuracy of engineered models, and their comparison with model structures. Models allowed us to investigate the distribution of charged groups on the surfaces of those proteins. Using developed models, we tend to create the primary attempt in correlating the structure with the thermal stability, and to reveal intramolecular tunnels as potential determinants of oxygen resistance.

\section{Methodology:}

\section{Mapping Intramolecular tunnels}

Intramolecular tunnels are of interest as potential pathways for entry of inhibitors like oxygen gas to the active sites of hydrogenases. Web-service MOLE online 2.0 [3] was used for investigation of intramolecular tunnels. Firstly hydrogenase from D. vulgaris Miyazaki F (PDB-entry ${ }_{1} \mathrm{H} 2 \mathrm{~A}$ ) was studied. The start line for tunnel searching was outlined by three amino acid residues annotated as active site in catalytic site Atlas information [4] consistent with the service choice. These residues were Glu L34 and Cys L546 within the massive fractional monetary unit and Thr S18 in the small subunit, being conservative for studied hydrogenases. The geometric parameters were configured to default values: minimal tunnel radius-1.2 $\mathrm{A}^{\circ}$ (which is giant Van der Waals radius of oxygen) and probe radius for surface construction $3 \mathrm{~A}{ }^{\circ}$. Place to begin was near the active site. Due to that, corresponding residues from template and models were selected for tunnel analysis, i.e., A Thr17, B Glu1014, and B Cys1555 for the template; S Thr17, L Cys555 and L Glu12 for models of HydSL from C. reinhardtii; and S Thr18, L Cys 607 and L Glu 19 for HydSL from C. vulgaris.

\section{Mapping molecular surfaces}

For mapping hydrophobic regions, hydrophobic residues of (Val, Leu, Ile, Met, Ala, Trp, and Phe) were identified and colored on molecular surfaces of the hydrogenases. For mapping charged regions, calculation of static potential in AMBER03 field [5] by Particle Mesh Ewald (PME) methodology [6] was administered in YASARA Structure at completely different $\mathrm{pH}$ values: 5, 7, and 9.

\section{Super positioning of the HydSL proteins}

Root-mean-square deviation (RMSD) of backbone atoms between models and between models and templates and between templates was calculated in Super Pose Version 1.0 [7]. For comparison, the same calculations were made with template structure (PDB-entry ${ }_{3} \mathrm{MYR}$ ) and with X-ray structure of hydrogenase from Desulfovibrio vulgaris Miyazaki F (PDB-entry 


\section{BIOINFORMATION}

Discovery at the interface of physical and biological sciences

\section{Open access}

$\left.{ }_{1} \mathrm{H} 2 \mathrm{~A}\right)$ that was chosen as reference thermolabile enzyme.

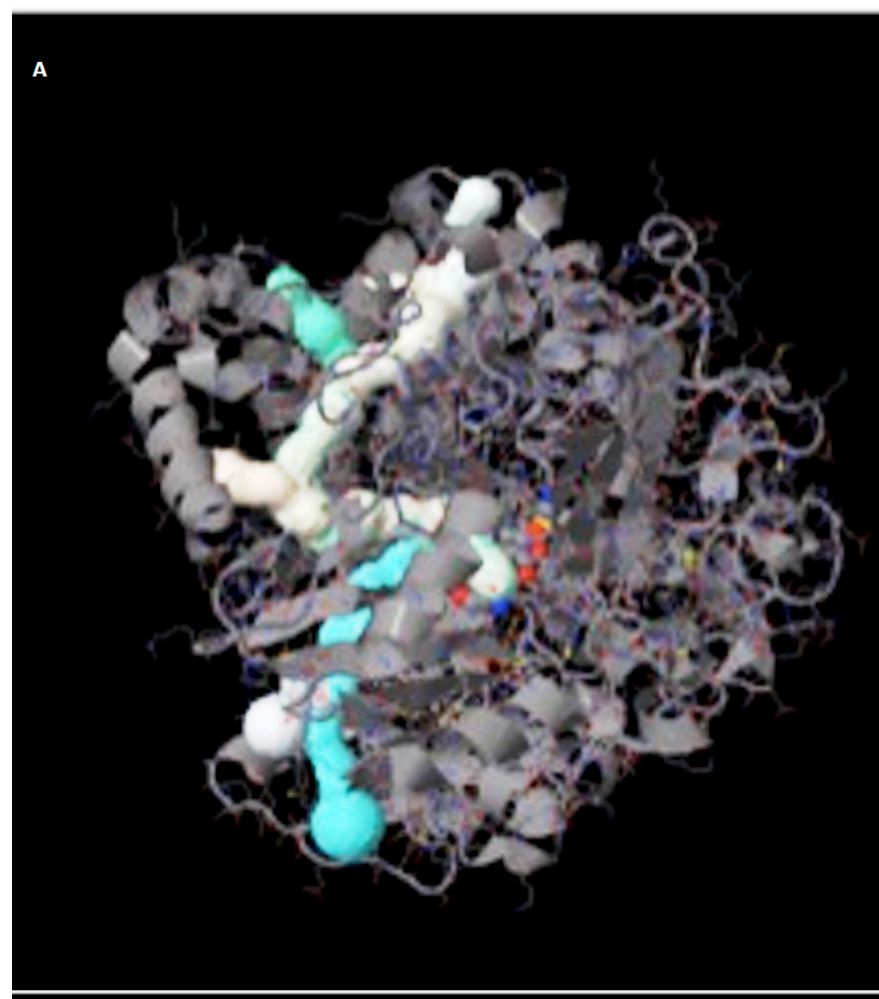

B

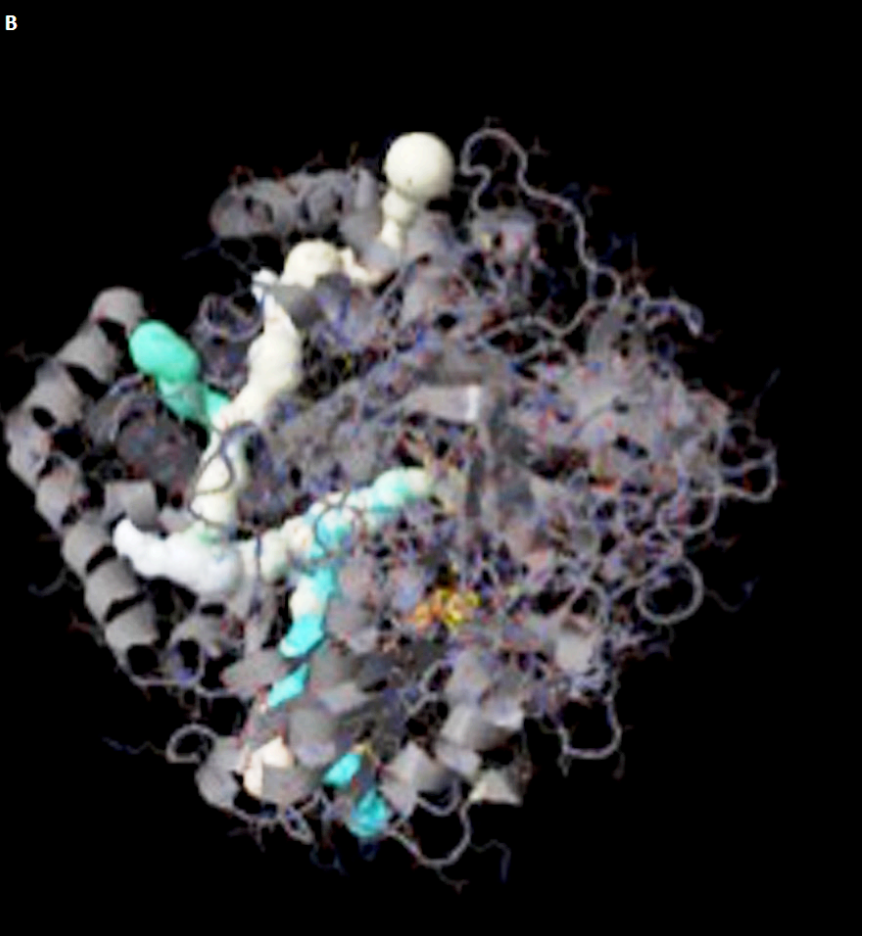

Figure 2: $(a, b)$ : Models of $C$. reinhardtii hydrogenase tunnels: two tunnels joined into one. The green, cyan and white colored coils are showing the hydrogenase tunnels assumed to be connecting all the active sites of HydSL enzyme. The presence of tunnels is considered as the pathway that facilitates gas diffusion and substrate entry to the active site but can also promote hydrogenase inhibition, more the tunnels more the inhibition.
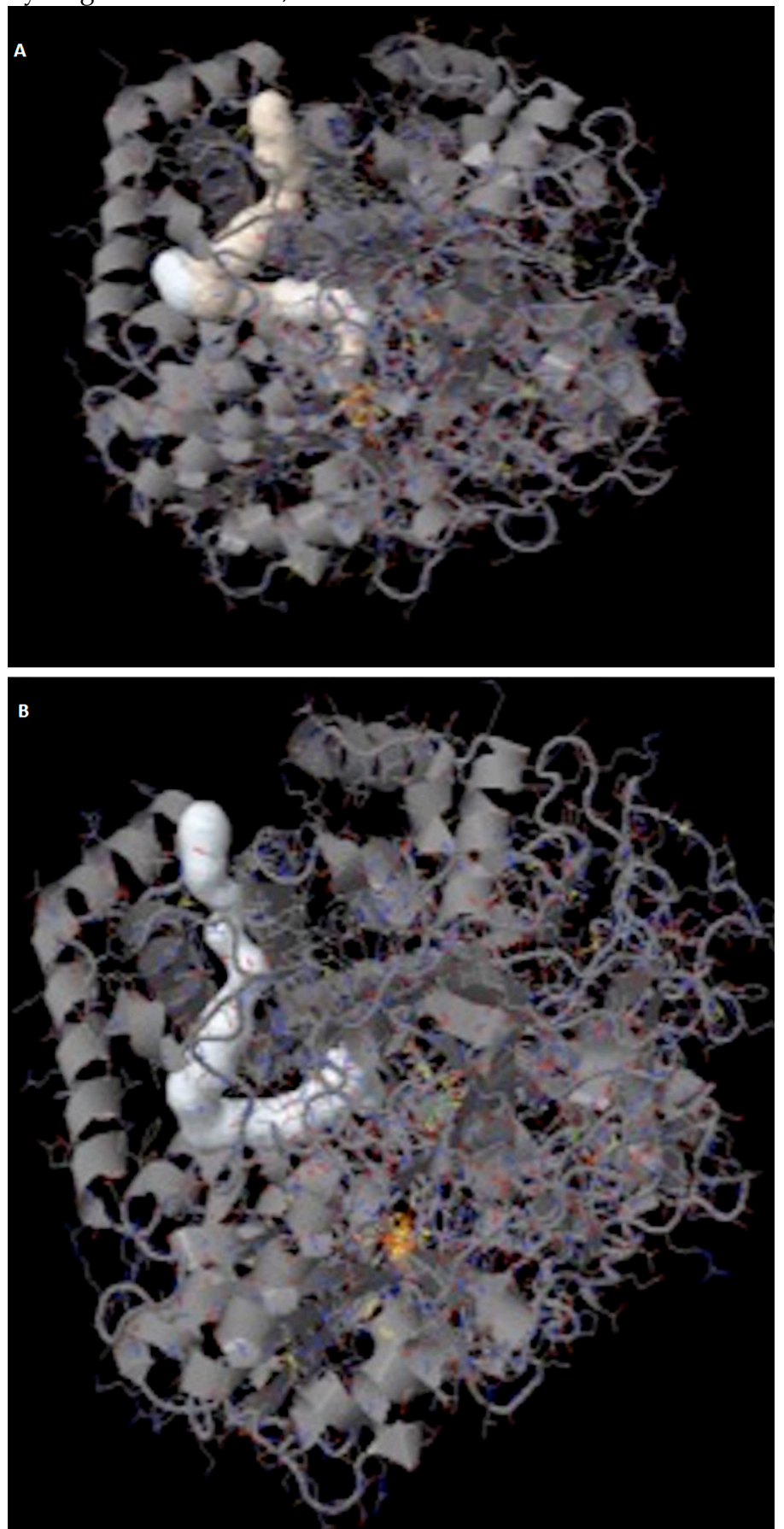

Figure 3: $(\mathrm{a}, \mathrm{b})$ Models of $C$. vulgaris hydrogenase tunnels; one tunnel almost like two tunnels. The cream and white colored coils are showing the hydrogenase tunnels assumed to be connecting all the active sites of HydSL enzyme. 


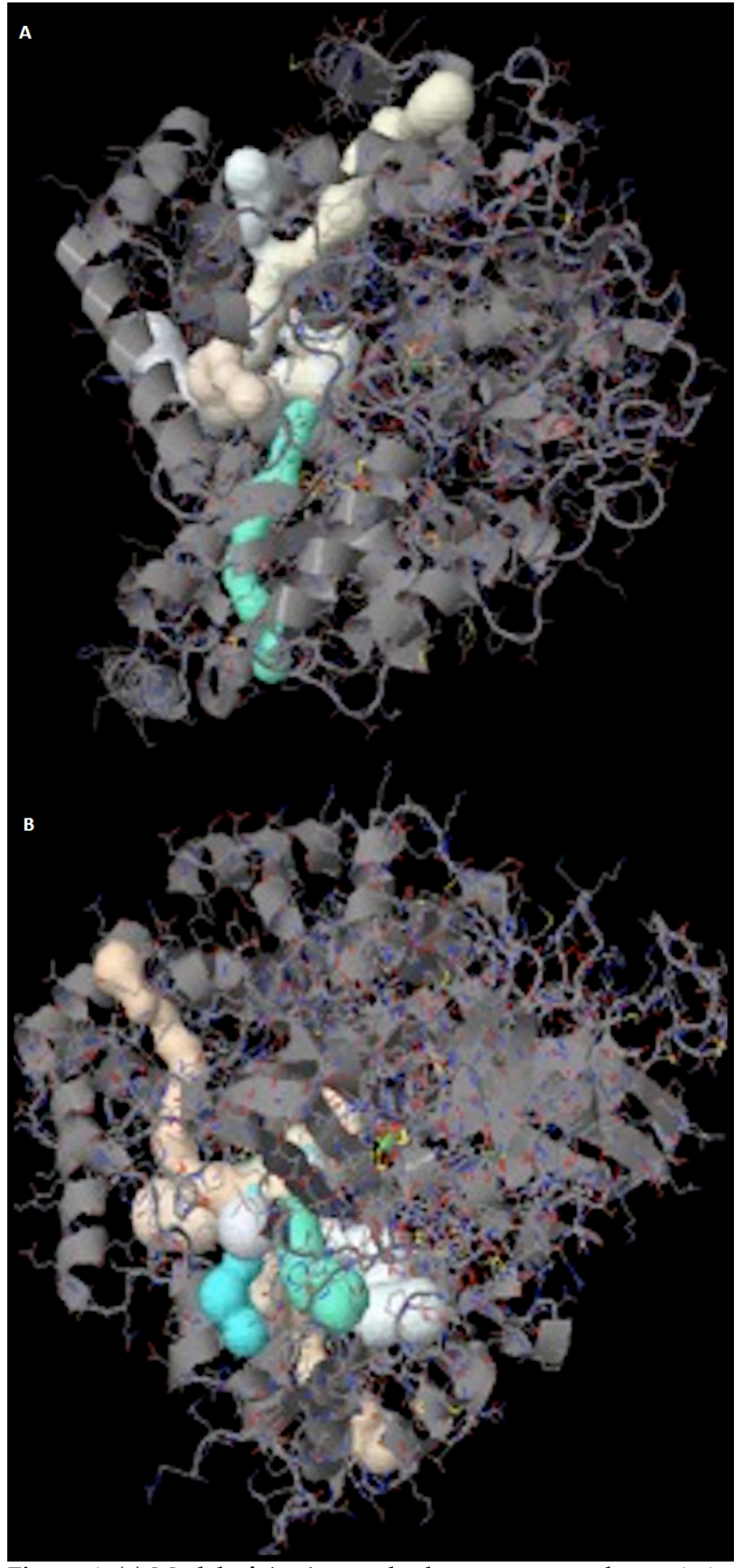

Figure 4: (a) Model of $A$. vinosum hydrogenase tunnels consisting of six tunnels, (b) Model of D. vulgaris hydrogenase tunnels consisting of six tunnels. The green, cyan, cream and white colored coils are showing the hydrogenase tunnels assumed to be connecting all the active sites of HydSL enzyme.

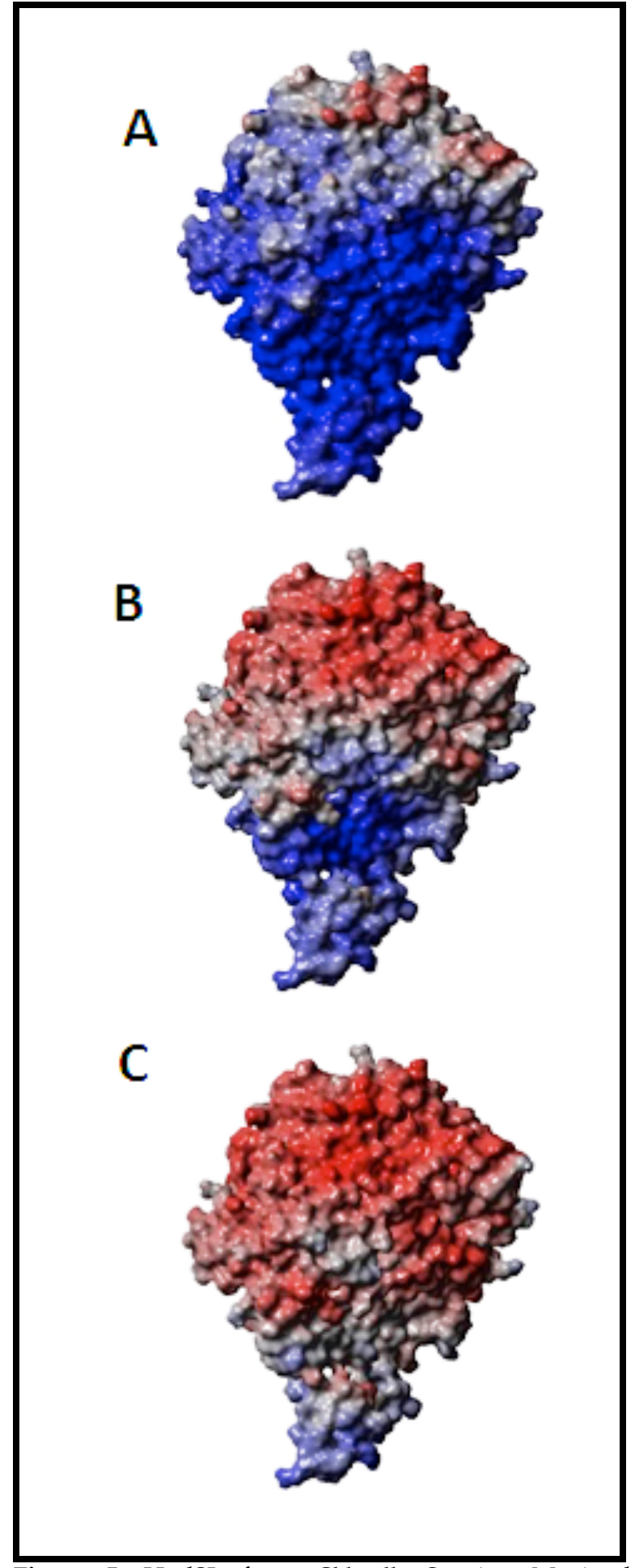

Figure 5: HydSL from Chlorella Species, Maximal potential (corresponding to the brightest color) was set to 250 $\mathrm{kcal} / \mathrm{mol}(1,046 \mathrm{~kJ} / \mathrm{mol})$. Positive potential areas are colored blue, negative-red, the neutral ones are gray. C-terminal parts of the small subunits are turned to the bottom. a) $\mathrm{pH}$ 5.0. b) $\mathrm{pH}$ 7.0.c) pH 9.0. 


\section{Open access}

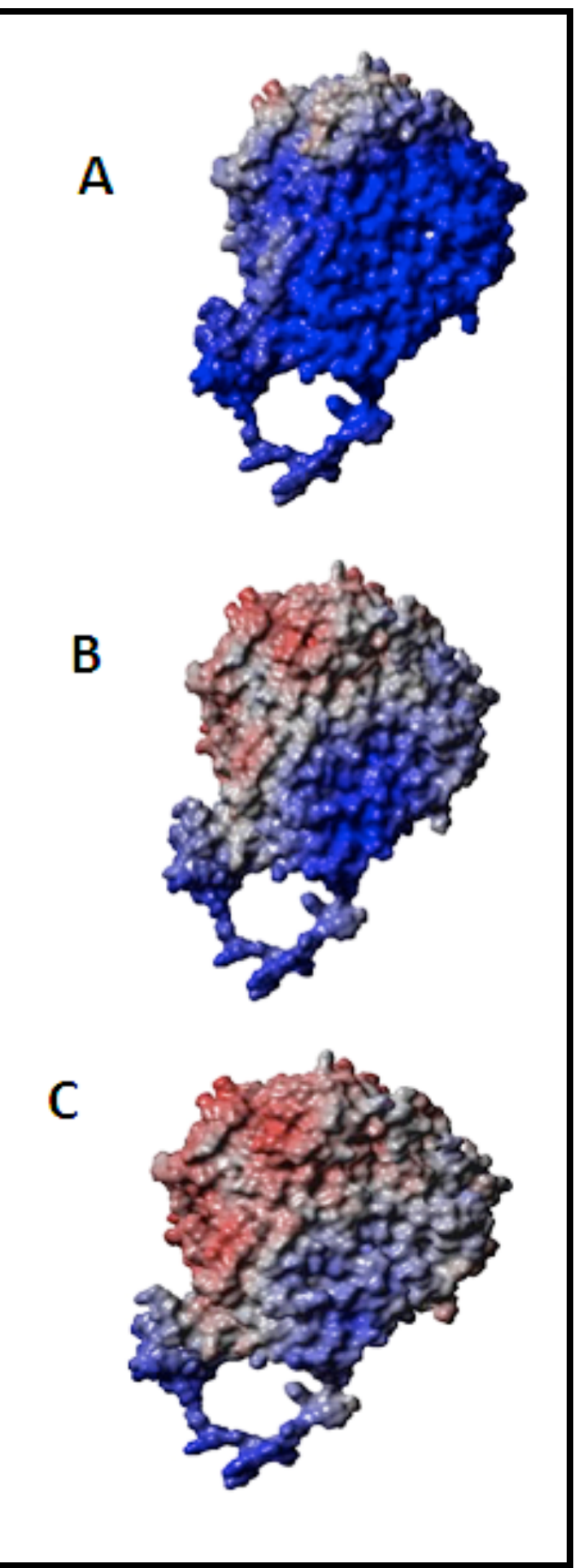

Figure 6: HydSL from Chlamydomonas Species, Maximal potential (corresponding to the brightest color) was set to 250

$\mathrm{kcal} / \mathrm{mol}(1,046 \mathrm{~kJ} / \mathrm{mol})$. Positive potential areas are colored blue, negative-red, the neutral ones are gray. C-terminal parts of the small subunits are turned to the bottom.a) $\mathrm{pH}$ 5.0. b) $\mathrm{pH}$ 7.0.c) $\mathrm{pH}$ 9.0 .
Results and Discussion:

Mapping intramolecular tunnels

The noteworthiness of tunnel structure of hydrogenases in their oxygen resistance is shown by multiple publications showing a high correlation between tunnel structure and oxygen resistance. Hydrogenases with low sensitivity to oxygen having constricted channels are an example [8]. Moreover, oxygen-sensitive hydrogenases of sulfate-reducing bacterium became insensitive once amino acids surrounding the tunnel were replaced by amino acids with large radical [9]. Additionally, there is no proof for the existence of $4 \mathrm{Fe}-3 \mathrm{~S}$ clusters (which was shown to be responsible for the oxygen sensitivity) in the small subunits attributable to the shortage of extra cysteines needed for amalgamate this cluster [10]. Also, there's no reason for the existence of selenocysteine residue within the active sites, which may well be a determinant of oxygen tolerance unless we are going to suppose a novel selenocysteine incorporation mechanism specific for these enzymes or the studied microorganism species.

In previous attempts researchers could not identify large amino acid residues specific for hydrogen-sensing hydrogenases in the corresponding positions of studied enzymes $[8,9]$. This is the first report of its kind in which mapping of the intramolecular tunnels in the four-hydrogenase enzymes disclosed potential variations between designed models and acknowledged structures. Models of $C$. reinhardtii hydrogenase had two tunnels join into one (Figure $2 \mathbf{a}, \mathbf{b}$ ), and models of $C$. vulgaris hydrogenase had one tunnel almost like two tunnels of $C$. reinhardtii (Figure $3 \mathbf{a}, \mathbf{b}$ ), whereas hydrogenases from $A$. vinosum (Figure 4 a) and $D$. vulgaris (Figure 4 b) Miyazaki F possessed six tunnels. Thus, there's a definite likelihood of the existence of a "bottleneck" in hydrogenases of $C$. reinhardtii and $C$. vulgaris creating tunnel mapping and thus oxygen diffusion not possible.

It should be noted that energy minimization has pointed to the impossibility of tunnel mapping; it implies that most of the time these enzyme tunnels are in closed state in the solution. Besides that, the tunnel structure depended on chosen methodology for subunit imposition that evidences their sensitivity to the geometry of mutual position of the subunits. For additional precise mapping of potential pathways for entry of inhibitors to the site, long molecular dynamics simulations in a particular solvent in the presence of oxygen molecules are needed.

\section{Mapping Electrostatic Potential}

The static potential maps were calculated by PME technique in the AMBER03 field of force (Figure $5 \& 6$ ). The hydrogenase of $C$. vulgaris possesses regions of positive potential that is sort of immune to $\mathrm{pH}$ alterations, within the $\mathrm{C}$-terminal region of tiny subunit and central part of the large one. Therefore, we are able to hypothesize that the optimum immobilisation agent for this protein would be a negatively charged molecule.

As for the hydrogenase of $C$. reinhardtii, it's more sensitive to hydrogen ion concentration shifts. That's why it looks that immobilisation agents for this protein ought to be varied reckoning on $\mathrm{pH}$. In acid or neutral conditions, negative molecules would be appropriate. 


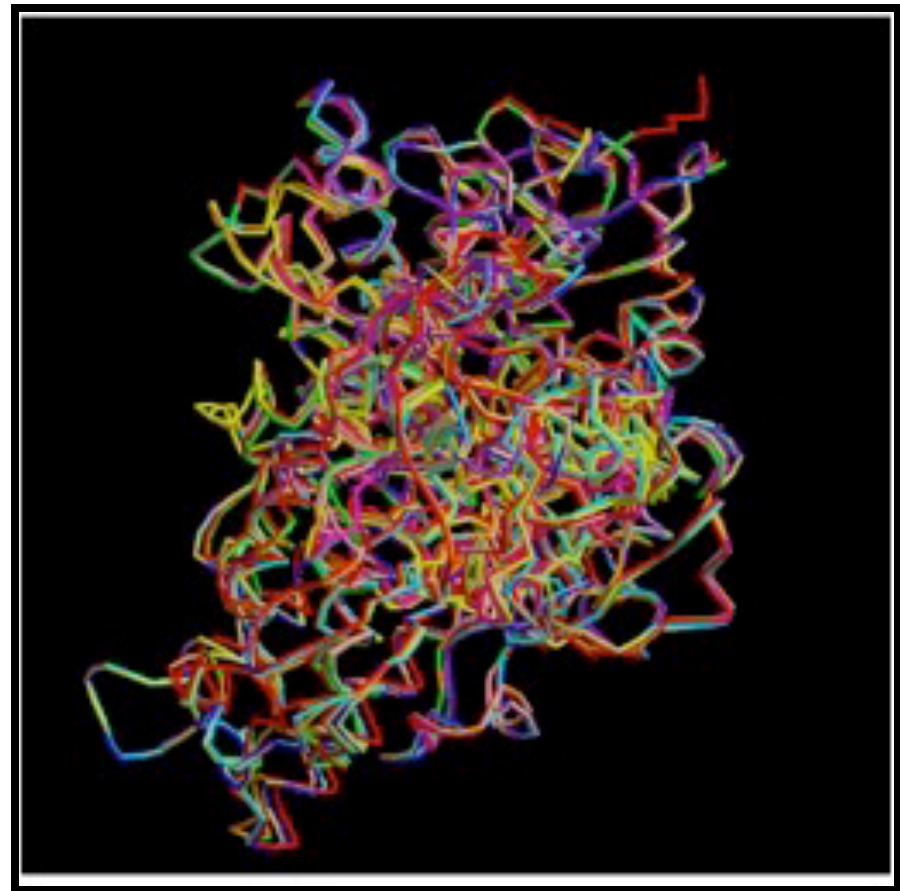

Figure 7: Super Pose output for multiple chain superposition using WebMole applet; superimposition was done of 8 chains of 3 MYR, 12 chains of ${ }_{2} \mathrm{FRV}$ and 2 chains of ${ }_{1} \mathrm{H} 2 \mathrm{~A}$ in backbone frame.

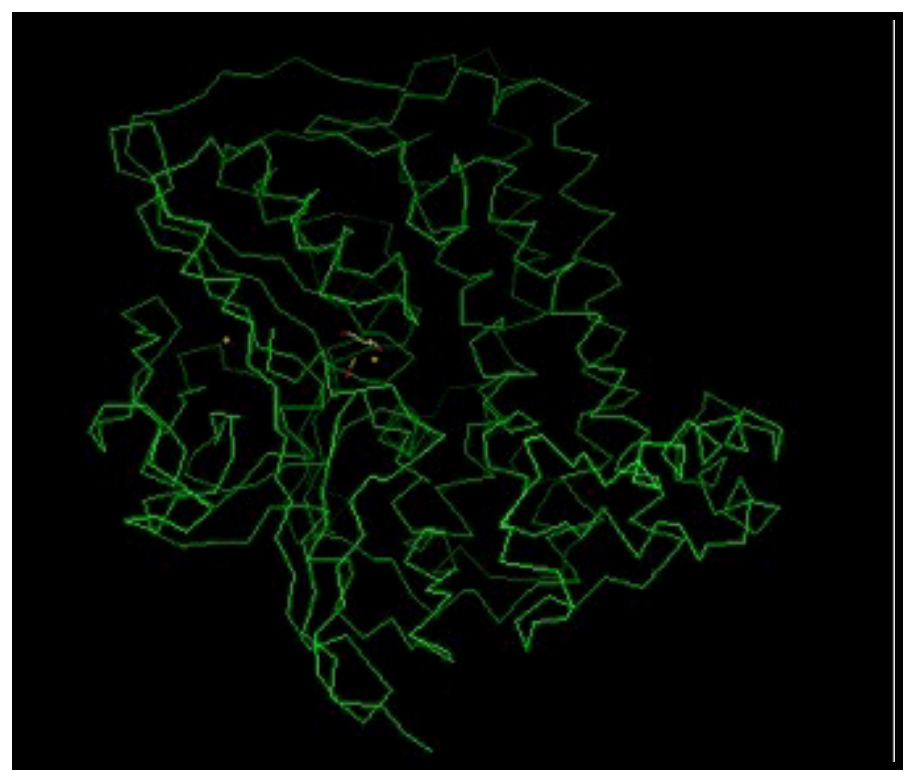

Figure 8: Super Pose output for multiple chain super-position using MolScript superposition applet showing atoms of the coordinating residues in a backbone frame and colored active sites supposedly connects all the tunnels.

\section{Super positioning of the HydSL proteins}

The Root-mean-square deviation (RMSD) of backbone atoms between models and between models and templates and between templates was calculated using Super Pose Version 1.0 (Figures 7 and 8). The combined RMSD value for all the templates and models was 1.284.RMSD value of less than $2 \AA$ is preferred as significant. RMSD $<2 \AA$ from the empirical structure depicts the model as a "Highly successful homology model" (Figure 9).

\begin{tabular}{|c|c|c|c|}
\hline \multicolumn{4}{|c|}{ Local RMSD - } \\
\hline & Carbons & $\frac{\text { Back }}{\text { Bone }}$ & Heavy All \\
\hline$\underline{\mathrm{RMSD}}$ & 1.144 & 1.147 & $1.284 \quad 1.284$ \\
\hline \multicolumn{4}{|c|}{ Global RMSD } \\
\hline & Carbons & $\frac{\text { Back }}{\text { Bone }}$ & Heavy All \\
\hline RMSD & 1.144 & 1.147 & $1.284 \quad 1.284$ \\
\hline
\end{tabular}

Figure 9: Super Pose output for multiple chain super-position depicting RMSD score of 1.284, showing a successful homology of the models and templates.

\section{Conclusions:}

Charged areas on hydrogenase molecule surfaces were mapped during this study, and it's shown that hydrogenases have hydrophobic regions, that might facilitate their incorporation into the membrane for interaction with photosynthetic electrontransport chain or at least coupling with hydrophobic components of proteins from the electron-transport chain. We also tried to seek out the explanations of oxygen resistance of studied hydrogenases within the structure of intramolecular tunnels. Superimposition of the templates and models showed RMSD value of 1.284, which showed significant successful homology of the HydSL. However, these tunnels behave like exceedingly labile structures, being sensitive to the mutual orientation of the subunits and to energy diminution simulation. Therefore, the next step of our study of them is mapping them in dynamic mode.

\section{Acknowledgements:}

Authors are thankful to DBT-SRF program for providing funding for the research and to Professor Rudi Ettrich, Academy of Sciences, Czech Republic for providing Yasara software for homology modeling.

\section{References:}

[1] Ogata $\mathrm{H}$ et al. Journal of the American Chemical Society, 2002 124(39): 11628 [PMID: 12296727]

[2] Vargas WA et al. Applied and environmental microbiology, 2011 77(6): 1990 [PMID: 21257809]

[3] Berka K et al. Nucleic acids research, 2012 40(W1): W222 [PMID: 22553366]

[4] Sillitoe I et al. Nucleic acids research, 2012 gks1211 [PMID: 23203873]

[5] Duan Y et al. Journal of computational chemistry, 2003 24(16): 1999 [PMID: 14531054] 
[6] Essmann U et al. Biophysical journal, 1999 76(4): 2081 [PMID: 10096903]

[7] Maiti R et al. Nucleic Acids Research, 200432 (Web Server issue), W590-W594.
[8] Buhrke T et al. Journal of Biological Chemistry, 2005 280(25): 23791 [PMID: 15849358]

[9] Leroux $F$ et al. Proceedings of the National Academy of Sciences, 2008 105(32): 11188 [PMID: 18685111]

[10] Volbeda A et al. Structure, 2013 21(1): 184 [PMID: 23260654]

Edited by P Kangueane Citation: Dixit et al. Bioinformation 12(3): 165-171 (2016) License statement: This is an Open Access article which permits unrestricted use, distribution, and reproduction in any medium provided the original work is properly credited. This is distributed under the terms of the Creative Commons Attribution License

\section{BIOMEDICAL

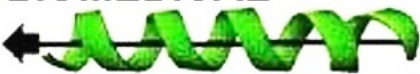 \\ INFORMATICS}

